\title{
Target chemotherapy of anti-CD147 antibody-labeled liposome encapsulated GSH-DXR conjugate on CD147 highly expressed carcinoma cells
}

\author{
HIROSHI MATSUDAIRA ${ }^{1,2}$, TADASHI ASAKURA ${ }^{1}$, KATSUHIKO AOKI $^{1}$, YASUYUKI SEARASHI ${ }^{2}$, \\ TOMOKAZU MATSUURA ${ }^{2}$, HISATO NAKAJIMA ${ }^{2}$, HISAO TAJIRI ${ }^{2}$ and KIYOSHI OHKAWA ${ }^{1}$ \\ Departments of ${ }^{1}$ Biochemistry and ${ }^{2}$ Internal Medicine Division of Gastroenterology \\ and Hepatology, Jikei University School of Medicine, Tokyo 105-8461, Japan
}

Received August 27, 2009; Accepted October 19, 2009

DOI: 10.3892/ijo_00000477

\begin{abstract}
It was confirmed that CD147 (Emmprin) was expressed on the cell surface of carcinoma cells. For the purpose of studying the efficacy of a CD147-targeting agent on CD147-expressing carcinoma cells, we investigated the effect of a conjugate of glutathione-doxorubicin (GSH-DXR) encapsulated in an anti-CD147 antibody-labeled liposome (aCD147ab-liposome) in terms of specific accumulation and cytotoxicity in CD147-expressing human carcinoma cells. Expression of CD147 was not observed in many normal human tissues. However, slight expression of CD147 in kidney, prostate and breast tissues was observed. By contrast, high-level expression of CD147 in all carcinoma cells such as A431, PC3 and Ishikawa cell lines was confirmed by fluorescent microscopy and Western blot analysis. Specific accumulation of the aCD147ab-liposome in the abovedescribed CD147-expressing cells was observed. GSH-DXR encapsulated in an aCD147ab-liposome expressed specific cytotoxicity against these carcinoma cells. These results suggested that target chemotherapy of GSH-DXR encapsulated in an aCD147ab-liposome on CD147-expressing carcinoma cells was effective.
\end{abstract}

\section{Introduction}

Conjugates of monoclonal antibodies (MAbs) with drugs or toxins have been investigated for many years as a potential approach to delivering these agents more specifically to cancers (1). For example, rituximab, a chimeric monoclonal antibody targeted against the pan-B-cell marker CD20, was the first monoclonal antibody to be approved for therapeutic

Correspondence to: Dr Hiroshi Matsudaira, Department of Internal Medicine Division of Gastroenterology and Hepatology, Jikei University School of Medicine, 3-25-8 Nishi-shinbashi, Minato-ku, Tokyo 105-8461, Japan

E-mail: hi-matz48@jikei.ac.jp

Key words: CD147 (Emmprin), immuno-liposome, target chemotherapy use (2-4). Treatment with rituximab at standard weekly dosing is effective in more than $50 \%$ of patients with relapsed or refractory CD20-positive follicular non-Hodgkin's lymphoma (5). Moreover, drug-MAb conjugates are currently under development for the treatment of various solid tumors (6).

Degradation of the basement membrane by matrix metalloproteinases (MMPs) is one of the most critical steps in the various stages of tumor disease progression, including tumor angiogenesis, tumor growth, as well as local invasion and subsequent distant metastasis (7-9). Extracellular matrix MMP, a family of zinc-dependent proteolytic enzymes, plays a central role in these processes, due to the ability to break down basement membranes and most extracellular matrix (ECM) components. Extracellular matrix MMP inducer (EMMPRIN, also known as CD147 or basigin) is a $55-\mathrm{kDa}$ molecule that is found on the surface of tumor cells and upregulates the expression of MMPs in surrounding fibroblasts and endothelial cells to enhance the invasiveness of cancer cells (10). Involvement of tumor-derived EMMPRIN in tumor angiogenesis through stimulation of MMPs and VEGF production was demonstrated by Tang et al (11), suggesting that CD147 could represent a key molecule in tumor cell invasion and metastasis. To assess its putative role as a target for anticancer therapies, Riethdorf et al (12), analyzed the overall incidence of CD147 expression in a wide spectrum of normal and tumor tissues. They showed that CD147 expression was detected frequently in the vast majority of human malignancies and also in a subset of benign tumors. Nevertheless, there are some very interesting differences both in the intensity and distribution of CD147 staining among different malignant tumors as well as benign lesions (12). It was predicted that CD147 has the potential to serve as a target for anti-tumor therapy.

A murine monoclonal antibody (MoAb 12C3) that is specific to human ovarian carcinoma was generated by immunizing mice with a human ovarian germinoma cell line (JOHYC-2) in a previous study by us (13). A single-phage clone was purified by immunostaining with MoAb 12C3 among the phages selected from either the human ovarian carcinoma (SKOV3) or colon carcinoma cDNA libraries, termed SKOV3-1 and colon-1, respectively. Based on the selected DNA sequences of the phage, a homology search 
using an amino acid sequence with the BLAST program was performed and revealed that SKOV3-1 and Colon-1 matched the 17th-174th and the 17th-149th amino acid residues, respectively, which were on the extracellular region of CD147 (14).

On the other hand, a recent study revealed that a conjugate of doxorubicin (DXR) with glutathione (GSH) (GSH-DXR) potently induced apoptosis in rat hepatoma AH66 cells relative to DXR (15-17). The same study also showed that GSH-DXR inhibited GST activity and suppressed GST P1 mRNA, but DXR did not, indicating that inhibition of the enzyme makes an important contribution to manifestation of potent GSH-DXR cytotoxicity against AH66 cells $(18,19)$. Therefore, it was predicted that the disappearance of GST P1-1 enzymatic activity did not suppress JNK activity in spite of binding to the JNK molecule and potently inducing apoptosis.

In the present study, we investigated the therapeutic efficacy of the aCD147ab-DXR conjugate for the treatment of several cancer cell lines. Moreover, GSH-DXR encapsulated in the aCD147ab-liposome was also examined for its specific cytotoxicity against these carcinoma cells.

\section{Materials and methods}

Materials. DXR was obtained from Kyowa Hakko Kogyo (Tokyo, Japan). GSH, MTT, 5-bromo-4-chloro-3-indolyl phosphate and nitro blue tetrazolium tablet (BCIP/NBT), Mission lentiviral short hairpin RNA (shRNA) for human CD147, anti-mouse IgG-alkaline phosphatase conjugate and anti-mouse IgG-FITC conjugate were obtained from SigmaAldrich Japan (Tokyo, Japan). Dowex 50W x8 and glutaraldehyde were purchased from Nakarai Tesque (Kyoto, Japan). Distearoyl-phosphatidylethanolamine-polyethylene glycolmaleimide (DSPE-PEG-MAL), DSPE-PEG-activated carboxylic acid (PE-PEG-NHS) and Coatsome (EL-01-A) were obtained from NOF corporation (Tokyo, Japan). Both lipids were contained PEG of $2 \mathrm{kDa}$. Vector of pQBI25-GFP was obtained from Wako chemicals (Tokyo, Japan). All other chemicals were of analytical grade.

Preparation of anti CD147 antibody. Murine anti-human CD147 monoclonal antibody (aCD147ab, MoAb 12C3) that is specific to human ovarian carcinoma was generated by immunizing mice with a human ovarian germinoma cell line (JOHYC-2) in our study (13).

Cell lines. Human epitherial carcinoma cell line, A431, human placental carcinoma cell line, Ishikawa, and human prostate carcinoma cell line, PC3 were cultured with RPMI-1640 containing $10 \%$ heat inactivated fetal bovine serum (growth medium) under conventional conditions.

Knockdown of CD147 expression in PC3 cells. Plasmid of shRNA for human CD147 $(0.1 \mu \mathrm{g}$ of plasmid in $10 \mathrm{~cm}$ dish $)$ was transfected into PC3 cells using the FuGENE6 transfection reagent (Roche). The transfectants were selected by treatment with puromycin. A permanent CD147-suppressive clone (PC3/CD147-KD) was obtained.
Conjugation of DXR with GSH. GSH-DXR was prepared as described previously (16-19). In brief, the combination of $1 \mathrm{mg}$ of each GSH and $0.5 \mathrm{mg}$ of DXR in $0.5 \mathrm{ml}$ of $0.15 \mathrm{M} \mathrm{NaCl}$ containing $0.1 \%$ glutaraldehyde was incubated at room temperature for $30 \mathrm{~min}$. After incubation, GSH-DXR was separated from GSH and DXR using Dowex $50 \mathrm{~W}$ x8 $\left(\mathrm{H}^{+}\right.$ form, $5 \times 15 \mathrm{~mm}$ ). The obtained GSH-DXR was filtersterilized by a $0.45-\mu \mathrm{m}$ syringe filter (Corning Coster, Tokyo Japan). The concentration of DXR was measured by absorbance at $495 \mathrm{~nm}$. A conjugation of aCD147ab and DXR was carried out in $0.1 \%$ glutaraldehyde at room temperature for 30 min. aCD147ab-DXR was purified by gel chromatography on Sephadex G-100.

Preparation of immuno ( $a C D 147 a b)$-liposome. One milligram of aCD147ab was mixed with $0.3 \mathrm{mg}$ of DSPE-PEG-NHS (activated carboxylic acid-terminal for $\mathrm{NH} 2$ group reaction) in $0.1 \mathrm{M}$ potassium phosphate buffer (K-PB) $(\mathrm{pH} 8.0)$ at room temperature (RT) overnight. One milligram of recombinant GFP was mixed with $0.3 \mathrm{mg}$ of DSPE-PEG-MAL (maleimideterminal for $\mathrm{SH}$ group reaction) in $0.1 \mathrm{M} \mathrm{K}-\mathrm{PB}(\mathrm{pH}$ 6.5) at RT overnight. After the reaction, excess non-reactive DSPE-PEGNHS and DSPE-PEG-MAL were quenched by incubation with $50 \mathrm{mM}$ Tris- $\mathrm{HCl}(\mathrm{pH} \mathrm{8.0)}$ and $2 \mathrm{mM}$ 2-mercaptoethanol, respectively, for $30 \mathrm{~min}$ at RT. 2-ME, DSPE-PEG and unconjugated antibody were removed by gel chromatography on Sephacryl S300HR. The resulting DSPE-PEG-aCD147ab and/or DSPE-PEG-GFP were mixed with $10 \mathrm{mg}$ of Coatsome EL-01-A in EtOH. Excess PBS was added to the mixture and centrifuged at 20,000 x g for $1 \mathrm{~h}$. The precipitate was suspended in PBS containing $100 \mu \mathrm{M}$ GSH-DXR and sterilized by passage through a $0.45-\mu \mathrm{m}$ sterile filter.

Expression of CD147 in human carcinoma cells (A431, Ishikawa and PC3). The cells were incubated with $1 \mu \mathrm{g} / \mathrm{ml}$ of aCD147ab for $1 \mathrm{~h}$. The cells were washed with PBS and were incubated with Alexa546-labeled anti-mouse IgG. The staining CD147 was observed by fluorescent microscopy.

Western blot analysis. CD147 in the cell extract with $1 \%$ Triton X-100 was separated by SDS-PAGE (10\% acrylamide) and analyzed by Western blot using aCD147ab as the primary antibody and ALPase-labeled anti-mouse IgG as the secondary antibody.

Immunoreaction of aCD147-labeled liposome. The cells were incubated with aCD147ab- and GFP-double labeled liposome for $2 \mathrm{~h}$. The cells were washed with PBS and were incubated with Alexa546-labeled anti-mouse IgG. The fluorescences of GFP and Alexa546 were detected by fluorescent microscopy.

Cytotoxicity of GSH-DXR-encapsulated immuno-liposome. Cytotoxicity of GSH-DXR-encapsulated immuno-liposome against A431, Ishikawa, PC3 and PC3/CD147-KD cells treated for $4 \mathrm{~h}$ was measured after $96 \mathrm{~h}$ by MTT assay. The cell death rate for aCD147ab-liposome/GSH-DXR $(0.1 \mu \mathrm{M})$ and mouse IgG-liposome/GSH-DXR $(0.1 \mu \mathrm{M})$ was expressed as drug-non treated control $(100 \%)$. 


\section{(A) Immunofluor escent microscopy}
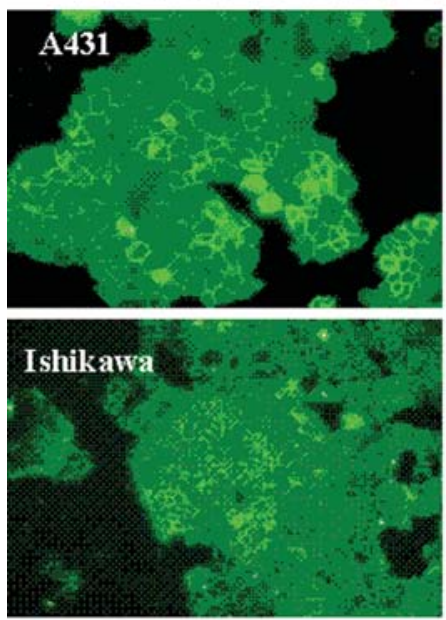
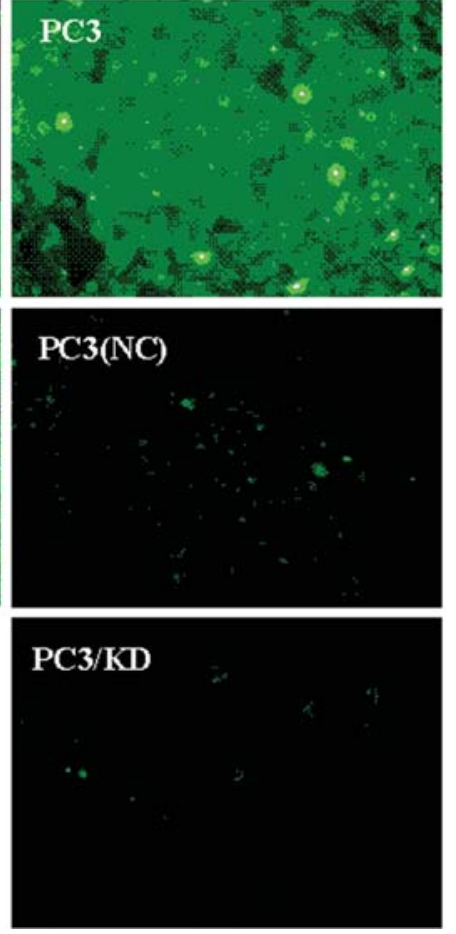

(B) Western blot

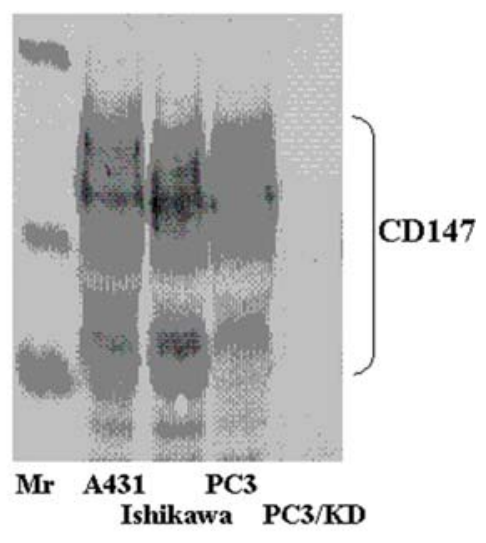

Figure 1. Expression of CD147 in A431 and Ishikawa, PC3 and PC3/KD cells detected by immunofluorescent microscopy (A) and by Western blot analysis (B). PC3 (NC), negative control (reaction with murine IgG instead of aCD147ab); PC3/KD, CD147-knockdown PC3 cells transfected shRNA for human CD147 (PC3/CD147-KD). Mr, protein marker.
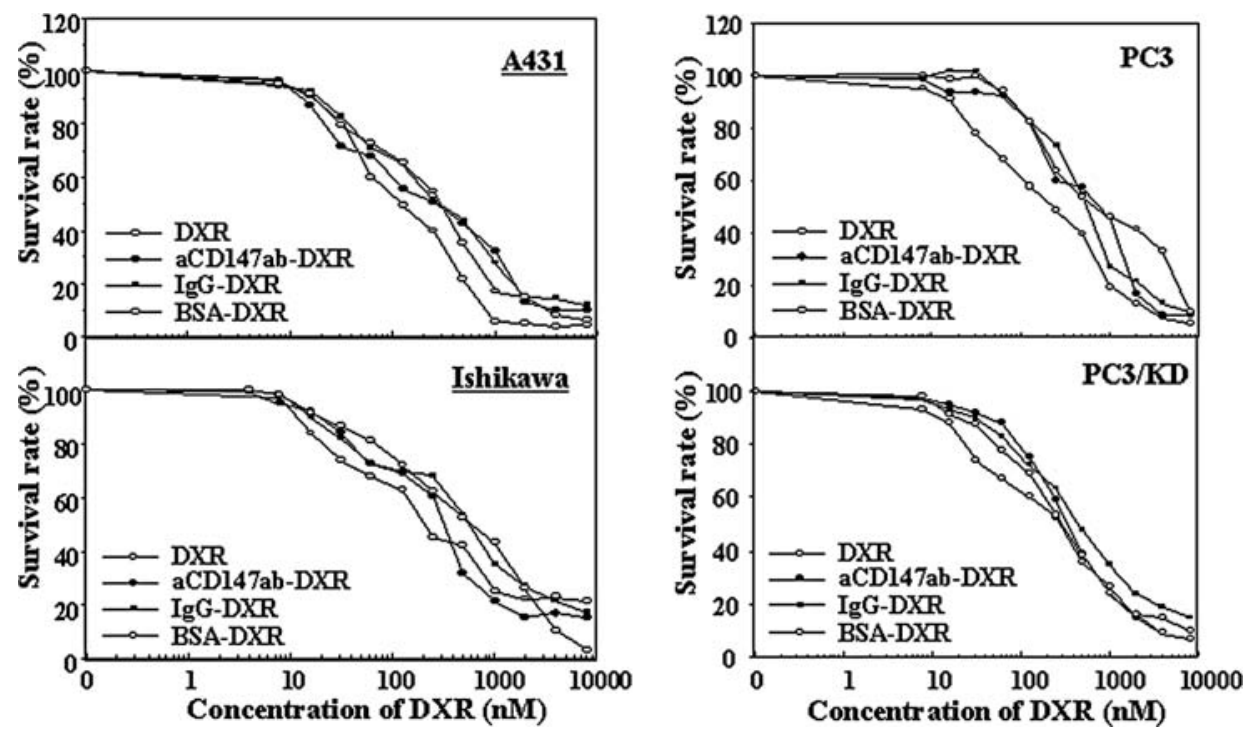

Figure 2. Cytotoxicity of aCD147ab-DXR and DXR conjugates against A431, Ishikawa, PC3 and PC3/CD147-KD cell lines. Concentration of aCD147abDXR was expressed as DXR concentration. After the conjugation of DXR was continuously exposed for $96 \mathrm{~h}$, cytotoxicity of the conjugate against each cell line was measured by MTT assay.

Protein determination. Protein concentration was assayed by a Bio-Rad protein assay kit using BSA as the standard.

\section{Results}

Expression of CD147 in A431, Ishikawa, PC3 and PC3/ CD147-KD cells with immunofluorescence and Western blot analysis. Representative profiles of immunofluorescence for CD147 are shown in Fig. 1A. Specific staining for CD147 was commonly observed in the cellular membrane of A431, Ishikawa and PC3 cells, whereas little immunofluorescent staining of the cellular membrane of CD147-knock down cells, PC3/CD147-KD, was observed. Moreover, the expression of CD147 (55-kDa) in A431, Ishikawa and PC3 cells at protein 

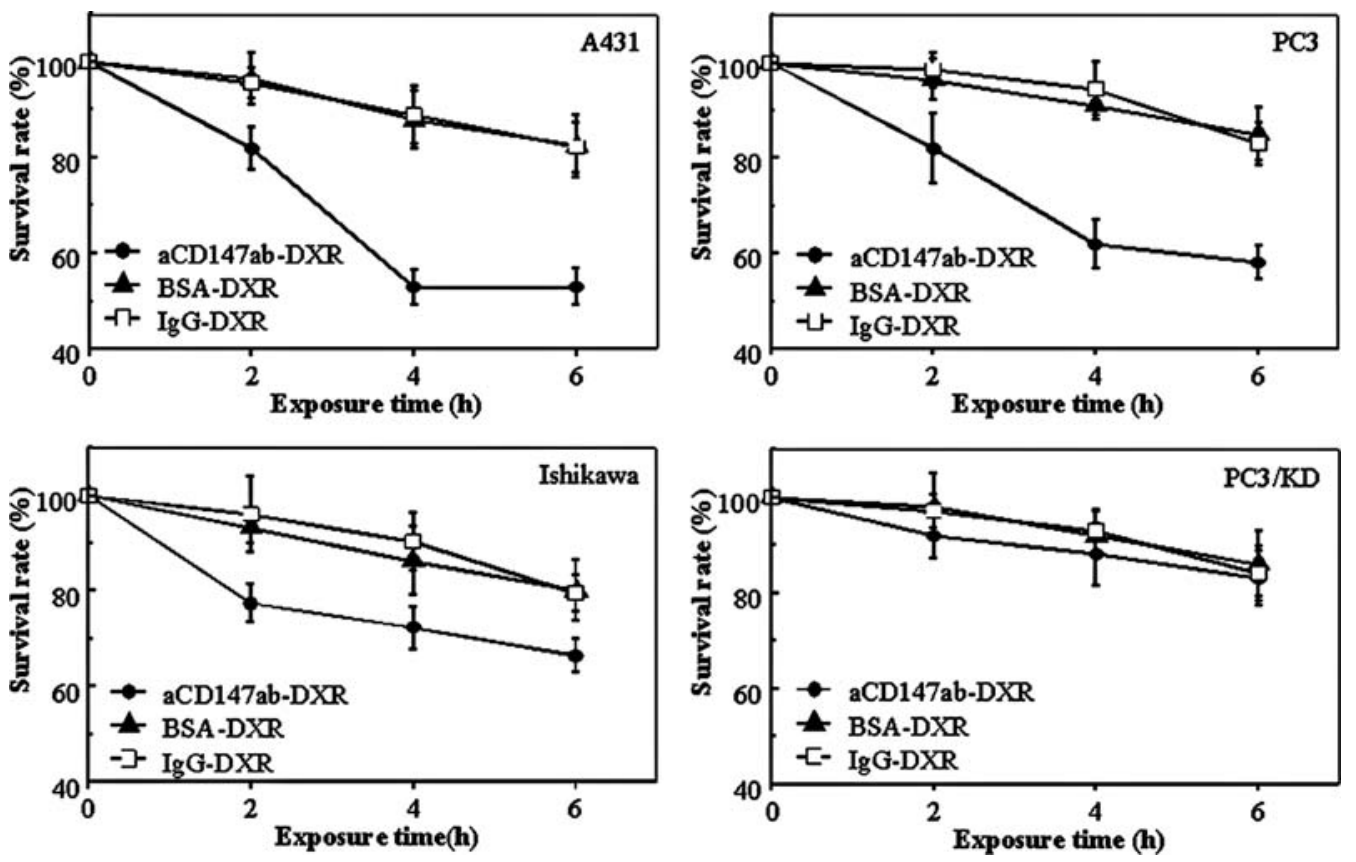

Figure 3. Survival rates of DXR conjugates with aCD147ab (aCD147ab-DXR), mouse IgG (IgG-DXR) and BSA (BSA-DXR) against A431, Ishikawa, PC3 and PC3/CD147-KD cell lines exposed for 2, 4 and $6 \mathrm{~h}$. MTT assay was carried out $96 \mathrm{~h}$ after treatment with DXR conjugates.

levels was observed by Western blot analysis using 12C3 as the primary antibody. Nevertheless, expression in PC3/ CD147-KD cells was not detected (Fig. 1B). The reason why broad and some bands of CD147 detected in this Western blot analysis was dependent upon different amount of glycosylation ratio of CD147.

Cytotoxicity of aCD147ab-DXR conjugates. We prepared the conjugate with DXR and aCD147ab, and examined the cytotoxicity. As shown in Fig. 2, after continuous exposure to aCD147ab-DXR, the A431, Ishikawa, PC3 and PC3/CD147KD cells exhibited the same degree of cytotoxicity in comparison to murine IgG-DXR and BSA-DXR used as the negative control.

As the $80 \%$ growth-inhibitory concentration value of these drugs was approximately $1 \mu \mathrm{M}$ of DXR-equivalent with these cell lines, the results of treatment conducted using the drugs at the concentration of $1 \mu \mathrm{M}$, with the cells exposed to the drugs for 2, 4 and $6 \mathrm{~h}$ after $96 \mathrm{~h}$ of incubation in drugfree medium are shown in Fig. 3. Cytotoxicity of aCD147abDXR was apparently higher than that of IgG-DXR or BSADXR. Moreover, the survival rates after exposure to the drug for $4 \mathrm{~h}$ were comparable among aCD147ab-DXR, IgG-DXR and BSA-DXR. The survival rate was 54,90 and $89 \%$, respectively, for A431 cells, 66, 86 and 91\%, respectively, for Ishikawa cells, 62, 90 and 93\% (51, 82 and 86\%), respectively, for PC3 cells, and 72, 80 and 82\%, respectively, for PC3/ CD147-KD cells. Accordingly, the efficacy of anti-cancer drugs targeting CD147 was demonstrated.

Accumulation of aCD147ab-DXR. When PC3 and PC3-KD cells were exposed to $1 \mu \mathrm{M}$ aCD147ab-DXR for $2 \mathrm{~h}$, the drug accumulated in the cell membrane of PC3 cells. As shown in Fig. 4, aCD147ab-DXR was combined and accumulated clearly at the CD147 discovery plasma membrane, and accumulation in PC3/CD147-KD cells was not observed.
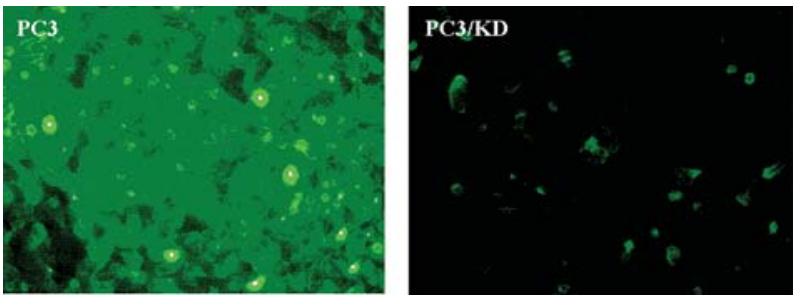

Figure 4. Accumulation of aCD147ab-DXR in PC3 and PC3/CD147-KD cells detected by immunofluorescent microscopy. After incubation with aCD147ab-DXR for $1 \mathrm{~h}$, the cells were washed with PBS and were incubated with Alexa546-labeled anti-mouse IgG. The fluorescences of Alexa546 were detected by fluorescent microscopy.

Accumulation of aCD147ab-liposome. After treatment of A431 cells with an aCD147ab-/GFP-double-labeled liposome for $2 \mathrm{~h}$, the cells were stained with Alexa546-labeled mouse IgG (secondary antibody for aCD147ab). The liposomes accumulated in the cells (Fig. 5A), but GFP-liposomes did not (data not shown). Moreover, co-treatment of the cells with the immuno-liposome and excess aCD147ab competitively suppressed accumulation of the liposome (Fig. 5B). Therefore, specific accumulation of the aCD147ab-liposome in CD147expressing cells was observed.

On the other hand, accumulation of the liposome in PC3 cells was observed by GFP-fluorescence. However, a small amount of the aCD147ab-liposome accumulated in PC3/ CD147-KD cells (Fig. 5C and D).

Cytotoxicity of aCD147ab-liposome against A431, Ishikawa, $P C 3$ and PC3/CD147-KD. Cytotoxicity of GSH-DXRencapsulated immuno-liposome against A431, Ishikawa, PC3 and PC3/CD147-KD cells treated for $4 \mathrm{~h}$ was measured after $96 \mathrm{~h}$. The cell death rate for the aCD147ab-liposome/GSHDXR $(0.1 \mu \mathrm{M})$ and mouse IgG-liposomes/GSH-DXR $(0.1 \mu \mathrm{M})$ 
(A) A431 +aCD147ab-liposome
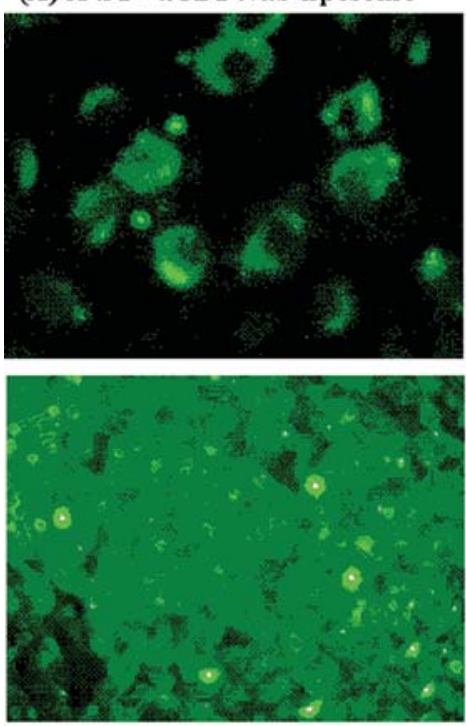

(C) PC3 +aCD147ab-liposome
(B) A431 + aCD147ab-liposome + excess aCD147ab
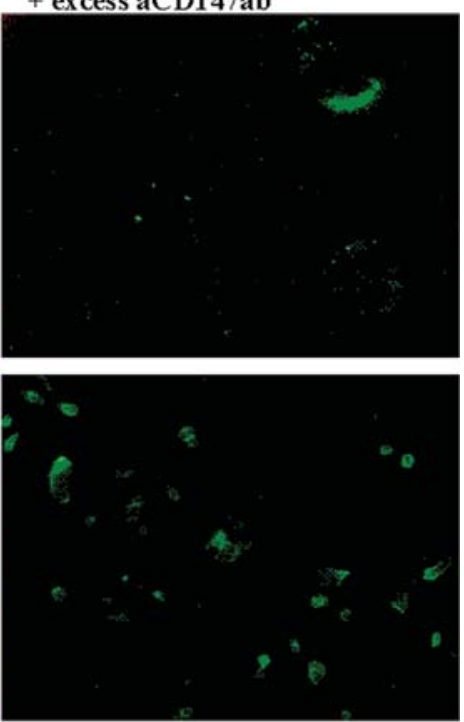

(D) PC3/KD +aCD147ab-liposome

Figure 5. Accumulation of aCD147ab-liposome in A431, PC3 and PC3/KD cells by fluorescent microscopy. (A) aCD147ab- and GFP-double labeled liposome in A431, (B) competitive inhibition of liposome-accumulation by excess aCD147ab in A431, (C) aCD147ab- and GFP-double labeled liposome in PC3, (D) aCD147ab- and GFP-double labeled liposome in PC3/KD. After treatment with aCD147ab- and GFP-double labeled liposome for 2 h, the cells were washed with PBS and were incubated with Alexa546-labeled anti-mouse IgG for $1 \mathrm{~h}$. The fluorescences of GFP and Alexa546 were detected by fluorescent microscopy.

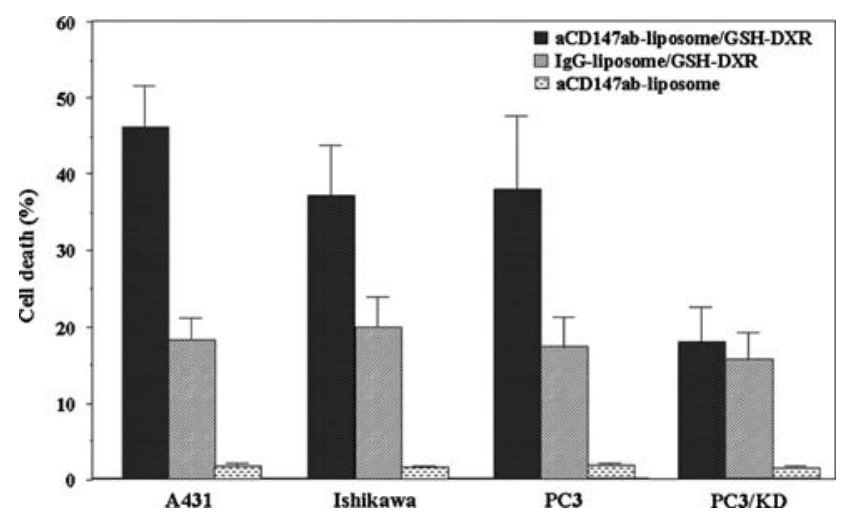

Figure 6. Cytotoxicity of aCD147ab-liposome/GSH-DXR for $4 \mathrm{~h}$ against A431, Ishikawa, PC3 and PC3/CD147-KD cells. MTT assay was carried out $96 \mathrm{~h}$ after treatment. The cell death rate for aCD147ab-liposome encapsulated GSH-DXR $(0.1 \mu \mathrm{M})$ (aCD147ab-liposome/GSH-DXR), mouse IgGliposome encapsulated GSH-DXR $(0.1 \mu \mathrm{M})$ (IgG-liposome/GSH-DXR) and aCD147ab-liposome without encapsulated-GSH-DXR (aCD147ab-liposome) was expressed as drug-non treated control (100\%).

was 46 and $18 \%$, respectively, for A431, 37 and 20\%, respectively, for Ishikawa, 38 and 17\%, respectively, for PC3, and 18 and 16\%, respectively, for PC3/CD147-KD (Fig. 6). On the other hand, little cytotoxic effect on these cells treated with aCD147ab-liposome without encapsulated-GSH-DXR was observed (less than 2.5\%). Moreover, leakage of GSHDXR from aCD147ab-liposome/GSH-DXR to culture medium for $4 \mathrm{~h}$-incubation was found in less than $2 \%$ of liposomal GSH-DXR (data not shown). These findings suggest that cytotoxicity of the aCD147ab-liposome/GSH-DXR was specifically related to the expression of CD147.

\section{Discussion}

Immunofluorescent study revealed a high level of CD147 protein expression in various carcinoma cells, but not in normal tissues. When taking into consideration that an anticancer drug acts at an early stage, it was predicted that CD147 would have sufficient potential for use in target therapies.

No significant difference in cytotoxicity was observed as a result of treating the cells with aCD147ab-DXR for $96 \mathrm{~h}$ compared with IgG-DXR or BSA-DXR. Nevertheless, even if the cells were exposed to aCD147ab-DXR for a short period of time (2-6 h), potent cytotoxicity of aCD147ab-DXR against carcinoma cells was observed compared with that of DXR. Moreover, it was demonstrated that the effect of aCD147ab-DXR on CD147-expressing cells specifically accumulated and exhibited potent cytotoxicity after only $2 \mathrm{~h}$ of exposure (Figs. 4-6). The CD147-negative normal cells did not show accumulation, and only weak cytotoxicity was observed against these cells. This anti-cancer drug targeting CD147 was shown to be effective even after short-term exposure. However, it was suggested that the same degree of cytotoxicity against all conjugates for $96 \mathrm{~h}$ exposure was caused by non-specific endocytotic uptake of drugs in 2-dimentional culture system.

Various physiological processes might be influenced by anti-EMMPRIN therapy (12), such as proliferation and differentiation of epithelial cells (20-22), fertilization (23), differentiation and activation of immune cells (24-26), integrinmediated adhesion of myocytes of the left heart ventricle to basement membrane components (27), selective transport processes in endothelial cells to maintain the blood-brainbarrier function (28), erythrocyte maturation (29) or wound 
healing (30). Accordingly, the side effects of target therapy using CD147 conjugates should be evaluated carefully.

We demonstrated in the previous study that GSH-DXR exhibited potent cytotoxicity compared to DXR (17-19). Here, the effect of drug-delivery of GSH-DXR on target chemotherapy was examined using the aCD147ab-liposome. The aCD147ab-liposome specifically accumulated in CD147expressing cells, but not in CD147-deficient PC3/CD147-KD cells. Moreover, the aCD147ab-liposome/GSH-DXR exhibited potent cytotoxicity against CD147-expressing cells. The drug was induced apoptotic cell death against these cells through caspase- 3 activation (data not shown). We also demonstrated that GSH-DXR reversed the response of MDR cells in which AH66/DR cells over-expressed Pgp and showed resistance to $10 \mu \mathrm{M}$ DXR (15-17). Therefore, it was predicted that target chemotherapy using GSH-DXR was effective on both drugsensitive and -resistant tumor cells.

In this study, we investigated the therapeutic efficacy of an aCD147ab-DXR conjugate for the treatment of several cancer cell lines. Moreover, the specific cytotoxicity of GSHDXR encapsulated in an aCD147ab-liposome against these carcinoma cells was also examined. Further study will attempt to confirm specific cytotoxicity in a three-dimensional cell culture system using the Radial-flow Bio-reactor.

\section{Acknowledgements}

This study was supported in part by The Jikei University Research Fund, by Grant-in-Aids for Scientific Research from Ministry of Health, Labour and Welfare, and by Grantin-Aids for R\&D Project on Molecular Imaging Equipment/ Development of Core Technology on Novel Molecular Imaging Probes for Malignant Tumor Detection/Development of Elemental Technology for Molecular Imaging Probes (FY2008-FY2009) from NEDO (New Energy and Industrial Technology Development Organization), Japan.

\section{References}

1. Trial PA, King HD and DubowchiK DM: Monoclonal antibody drug immunoconjugates for targeted treatment of cancer. Cancer Immunol Immunother 52: 328-337, 2003.

2. Anderson DR, Grillo-López A, Varns C, Chambers KS and Hanna N: Targeted anti-cancer therapy using rituximab, a chimaeric anti-CD20 antibody (IDEC-C2B8) in the treatment of non-Hodgkin's B-cell lymphoma. Biochem Soc Trans 25: 705-708, 1997.

3. Maloney DG, Grillo-López AJ, White CA, Bodkin D, Schilder RJ Neidhart JA, Janakiraman N, Foon KA, Liles TM, Dallaire BK, Wey K, Royston I, Davis T and Levy R: IDEC-C2B8 (Rituximab) anti-CD20 monoclonal antibody therapy in patients with relapsed low-grade non-Hodgkin's lymphoma. Blood 90: 2188-2195, 1997.

4. Maloney DG, Grillo-López AJ, Bodkin DJ, White CA, Liles TM, Royston I, Varns C, Rosenberg J and Levy R: IDEC-C2B8: results of a phase I multiple-dose trial in patients with relapsed non-Hodgkin's lymphoma. J Clin Oncol 15: 3266-3274, 1997.

5. Witziq TE, White CA, Wiseman GA, Gordon LI, Emmanouilides C, Raubitschek A, Janakiraman N, Gutheil J, Schilder RJ, Spies S, Silverman DH, Parker E and GrilloLópez AJ: Phase I/II trial of IDEC-Y2B8 radioimmunotherapy for treatment of relapsed or refractory CD20 (+) B-cell nonHodgkin's lymphoma. J Clin Oncol 17: 3793-3803, 1999.

6. Griffiths GL, Mattes MJ, Stein R, Govindan SV, Horak ID, Hansen HJ and Goldenberg DM: Cure of SCID mice bearing human B-lymphoma xenografts by an anti-CD74 antibodyanthracycline drug conjugate. Clin Cancer Res 9: 6567-6571, 2003 .
7. Nelson AR, Fingleton B, Rothenberg ML and Matrisian LM: Matrix metalloproteinases: biologic activity and clinical implications. J Clin Oncol 18: 1135-1149, 2000.

8. Fang J, Shing Y, Wiederschain D, Yan L, Butterfield C, Jackson G, Harper J, Tamvakopoulos G and Moses MA: Matrix metalloproteinase-2 is required for the switch to the angiogenic phenotype in a tumor model. Proc Natl Acad Sci USA 97: 3884-3889, 2000.

9. Bergers G, Brekken R, McMahon G, Vu TH, Itoh T, Tamaki K, Tanzawa K, Thorpe P, Itohara S, Werb Z and Hanahan D: Matrix metalloproteinase-9 triggers the angiogenic switch during carcinogenesis. Nat Cell Biol 2: 737-744, 2000.

10. Yan L, Zucker S and Toole BP: Roles of multifunctional glycoprotein, emmprin (basigin; CD147), in tumour progression. Thromb Haemost 93: 199-204, 2005.

11. Tang Y, Nakada MT, Kesavan P, McCabe F, Millar H, Rafferty P, Bugelski P and Yan L: Extracellular matrix metalloproteinase inducer stimulates tumor angiogenesis by elevating vascular endo-thelial cell growth factor and matrix metalloproteinases. Cancer Res 65: 3193-3199, 2005.

12. Riethdorf S, Reimers N, Assmann V, Kornfeld JW, Terracciano L, Sauter G and Pantel K: High incidence of EMMPRIN expression in human tumors. Int J Cancer 119: 1800-1810, 2006.

13. Yamada K, Ohkawa K and Joh K: Monoclonal antibody, Mab $12 \mathrm{C} 3$, is a sensitive immunohistochemical marker of early malignant change in epithelial ovarian tumors. Am J Clin Pathol 103: 288-294, 1995.

14. Ishibashi Y, Matsumoto T, Niwa M, Suzuki Y, Omura N, Hanyu N, Nakada K, Yanaga K, Yamada K, Ohkawa K, Kawakami $\mathrm{M}$ and Urashima M: CD147 and matrix metalloproteinase-2 protein expression as significant prognostic factors in esophageal squamous cell carcinoma. Cancer 101: 1994-2000, 2004.

15. Takahashi N, Asakura T and Ohkawa K: Pharmacokinetic analysis of protein-conjugated doxorubicin (DXR) and its degraded adducts in DXR-sensitive and -resistant rat hepatoma cells. Anti-Cancer Drugs 7: 687-696, 1996.

16. Asakura T, Takahashi N, Takada K, Inoue $\mathrm{T}$ and Ohkawa K: Drug conjugate of doxorubicin with glutathione is a potent reverser of multidrug resistance in rat hepatoma cells. AntiCancer Drugs 8: 199-203, 1997.

17. Asakura T, Sawai T, Hashidume Y, Ohkawa Y, Yokoyama S and Ohkawa K: Caspase-3 activation during doxorubicin conjugated with glutathione-mediated apoptosis. Br J Cancer 80: 711-715, 1999.

18. Asakura T, Ohkawa K, Takahashi N, Takada K, Inoue T and Yokoyama S: Glutathione-doxorubicin conjugate expresses potent cytotoxicity by a suppression of glutathione S-transferase activity: Comparison between doxorubicin-sensitive and-resistant rat hepatoma cells. Br J Cancer 76: 1333-1337, 1997.

19. Asakura T, Hashizume Y, Tashiro K, Searashi Y, Ohkawa K, Nishihira J, Sakai M and Shibasaki T: Suppression of GST-P by treatment with glutathione-doxorubicin conjugate induces potent apoptosis in rat hepatoma cells. Int J Cancer 94: 171-177, 2001.

20. De Castro R, Zhang Y, Guo H, Kataoka H, Gordon MK, Toole B and Biswas G: Human keratinocytes express EMMPRIN, an extracellular matrix metalloproteinase inducer. J Invest Dermatol 106: 1260-1265, 1996.

21. Chen X, Kanekura T and Kanzaki T: Expression of Basigin in human fetal, infantile and adult skin and in basal cell carcinoma. J Cutan Pathol 28: 184-190, 2001.

22. Chen X, Kanekura T, Tsuyama S, Murata F and Kanzaki T: Ultrastructural localization of basigin in normal human epidermis. Histochem Cell Biol 115: 465-470, 2001.

23. Kuno N, Kadomatsu K, Fan QW, Hagihara M, Senda T, Mizutani $S$ and Muramatsu T: Female sterility in mice lacking the basigin gene, which encodes a transmembrane glycoprotein belonging to the immunoglobulin superfamily. FEBS Lett 425: 191-194, 1998.

24. Koch C, Staffler G, Huttinger R, Hilgert I, Prager E, Cerny J, Steinlein P, Majdic O, Horejsí V and Stockinger H: T cell activation-associated epitopes of CD147 in regulation of $\mathrm{T}$ cell response, and their definition by antibody affinity and antigen density. Int Immunol 11: 777-786, 1999.

25. Kirsch AH, Diaz LA Jr, Bonish B, Antony PA and Fox DA: The pattern of expression of CD147/neurothelin during human T-cell ontogeny as defined by the monoclonal antibody 8D6. Tissue Antigens 50: 147-152, 1997. 
26. Zhu P, Ding J, Zhon J, Dong WJ, Fan CM and Chen ZN: Expression of CD147 on monocytes/macrophages in rheumatoid arthritis: its potential role in monocyte accumulation and matrix metalloproteinase production. Arthritis Res Ther 7: R1023-R1033, 2005.

27. Coker ML, Doscher MA, Thomas CV, Galis ZS and Spinale FG: Matrix metalloproteinase synthesis and expression in isolated LV myocyte preparations. Am J Physiol 277: H777-H787, 1999.

28. Sameshima T, Nabeshima K, Toole BP, Inoue T, Yokogami K, Nakano S, Ohi T and Wakisaka S: Correlation of emmprin expression in vascular endothelial cells with blood-brain-barrier function: a study using magnetic resonance imaging enhanced by Gd-DTPA and immunohistochemistry in brain tumors. Virchows Arch 442: 577-584, 2003.
29. Coste I, Gauchat JF, Wilson A, Izui S, Jeannin P, Delneste Y, MacDonald HR, Bonnefoy JY and Renno T: Unavailability of CD147 leads to selective erythrocyte trapping in the spleen. Blood 97: 3984-3988, 2001.

30. Gabison EE, Mourah S, Steinfels E, Yan L, Hoang-Xuan T, Watsky MA, De Wever B, Calvo F, Mauviel A and Menashi S: Differential expression of extracellular metalloproteinase inducer (CD147) in normal and ulcerated corneas: role in epitheliostromal interactions and matrix metalloproteinase induction. Am J Pathol 166: 209-219, 2005. 